\title{
Aceites de oliva vírgenes y refinados: Diferencias en componentes menores glicerídicos
}

\author{
Por M.C. Pérez-Camino, M.V. Ruiz-Méndez, \\ G. Márquez-Ruiz y M.C. Dobarganes
}

Instituto de la Grasa y sus Derivados (C.S.I.C.). Avda. Padre García Tejero 4. 41012 Sevilla. España.

\section{RESUMEN}

Aceites de oliva vírgenes y refinados: Diferencias en componentes menores glicerídicos.

En el presente trabajo se definen las principales diferencias en componentes menores glicerídicos entre aceites de oliva vírgenes y refinados. Para ello, se estudian las modificaciones que tienen lugar durante las distintas etapas del proceso de refinación, se comprueban las diferencias encontradas en dos series de aceites vírgenes y refinados $y$, finalmente, se analizan las modificaciones originadas durante el almacenamiento.

Los resultados demuestran que la ausencia de compuestos de polimerización es de gran valor para la caracterización de los aceites de oliva vírgenes, mientras que, la presencia de dímeros de triglicéridos y la elevada relación diglicéridos/ácidos grasos son las características principales de los aceites refinados.

PALABRAS-CLAVE: Aceite de oliva refinado - Aceite de oliva virgen Componentes menores glicerídicos - Cromatografía de exclusión.

\section{SUMMARY}

Virgin and refined olive oils: Differences in glyceridic minor compounds.

This paper defines the main differences in glyceridic minor compounds between virgin and refined olive oils. Modifications during the steps of the refining process are studied and compared with differences found in two series of virgin and refined oils and those during storage.

The results demonstrate that the absence of polymerized compounds is the most useful parameter for the characterization of virgin oils while the presence of dimeric triglycerides and a high ratio diglycerides/fatty acids are those for refined oils.

KEY-WORDS: Glyceridic minor compounds - Refined olive oil - Size exclusion chromatography - Virgin olive oil.

\section{INTRODUCCION}

Como es conocido, el aceite de oliva procede de un fruto (Olea europaea) y se consume como virgen, refinado o mezcla de ambos, a diferencia de los aceites de semillas, que se consumen después de ser refinados. Tiene, por ello, un gran interés establecer parámetros que permitan caracterizar los distintos tipos de aceites de oliva que pueden ser comercializados.

Los parámetros fisicoquímicos más diferenciadores de ambos tipos de aceites de oliva son los valores de las extinciones específicas a 232 y $270 \mathrm{~nm}$ (Boletín Oficial del Estado, 1983; Diario Oficial de las Comunidades Europeas, 1991), más elevadas en los aceites refinados como consecuencia de la formación de dienos y trienos conjugados durante el proceso de refinación. Las diferencias no son, sin embargo, suficientes para caracterizar mezclas de vírgen-refinado, sobre todo cuando contienen una proporción baja de aceite refinado en aceite virgen.

Existe un considerable número de estudios dirigidos a conocer las propiedades y características del aceite de oliva (Kiritsakis, 1991; Faur, 1989a y 1989b) y las modificaciones que tienen lugar durante la refinación tanto de la fracción glicerídica mayoritaria (Taponeco y Giaconi, 1970; Gracián y Mancha, 1971; Amelotti, 1987) como en la fracción insaponificable (Johansson, 1979; Kochchar, 1983). Los trabajos de mayor interés son aquellos que se refieren a la detección de compuestos específicos que se originan como consecuencia de alguna de las fases del proceso de refinación y que, al mismo tiempo, estén prácticamente ausentes en los aceites vírgenes (Eder, 1982; Gomes y Catalano, 1988; Lanzón et al., 1989). Su presencia, por tanto, en cantidades significativas sería indicativa de la existencia de aceite refinado en mezcla con aceite virgen.

En este trabajo se definen las principales diferencias en componentes menores glicerídicos entre aceites de oliva vírgenes y refinados. Para ello, se estudian las modificaciones que tienen lugar durante las distintas etapas del proceso de refinación, se comprueban las diferencias encontradas en dos series de aceites vírgenes y refinados y, finalmente, se analizan las modificaciones originadas durante el almacenamiento prolongado de aceites de oliva vírgenes.

\section{PARTE EXPERIMENTAL}

\subsection{Muestras}

Para el desarrollo de este estudio se ha dispuesto de un variado número de aceites de oliva vírgenes y refinados clasificados en cuatro grupos:

- Dos aceites vírgenes de distinta calidad inicial que se someten a refinación en las condiciones que se indican con 
posterioridad. Las características físico-químicas de los mismos se recogen en la Tabla I.

- 15 muestras de aceites de oliva vírgenes procedentes de diferentes cooperativas.

- 15 muestras de aceites de oliva refinados procedentes de distintas industrias.

- 12 muestras de aceites de oliva de calidad obtenidas a partir de tres variedades de aceitunas (picuda, picual y marteña), con dos grados de madurez y elaborados mediante los sistemas de prensa y centrifugación (F.A.O. et al., 1975). Estos aceites son evaluados inmediatamente después de su obtención, así como después de un año de conservación a temperatura ambiente en envases de cloruro de polivinilo (PVC) de $1 \mathrm{~L}$ de capacidad.

Tabla I

Características físico-químicas de los aceites antes de ser refinados

\begin{tabular}{|c|c|c|}
\hline & MUESTRA 1 & MUESTRA 2 \\
\hline $\begin{array}{l}\text { Acidez } \\
\text { (\% Oleico) }\end{array}$ & 6.38 & 1.17 \\
\hline $\begin{array}{l}\text { Estabilidad } \\
\text { (h) }\end{array}$ & 8.3 & 29.4 \\
\hline $\mathbf{K}_{270}$ & 0.38 & 0.17 \\
\hline $\begin{array}{l}\text { Insaponificable } \\
(\%)\end{array}$ & 0.98 & 1.40 \\
\hline \multicolumn{3}{|l|}{$\begin{array}{l}\text { Acidos Grasos } \\
\text { Mayoritarios }\end{array}$} \\
\hline $\mathrm{C}_{16: 0}$ & 13.2 & 11.0 \\
\hline $\mathrm{C}_{16: 1}$ & 0.8 & 0.9 \\
\hline $\mathrm{C}_{18: 0}$ & 1.9 & 2.1 \\
\hline $\mathrm{C}_{18: 1}$ & 71.2 & 78.5 \\
\hline $\mathrm{C}_{18: 2}$ & 11.7 & 6.4 \\
\hline
\end{tabular}

\subsection{Condiciones de refinación}

Los aceites vírgenes, cuyas características se recogen en la Tabla I, se refinan en sistema de laboratorio, en las condiciones que se resumen a continuación:

\section{a) Refinación química}

Muestras de $250 \mathrm{~g}$ se calientan a $80^{\circ} \mathrm{C}$ y se neutralizan con una solución de sosa de $18^{\circ} \mathrm{Bé}$. La cantidad de sosa utilizada fue un $10 \%$ de exceso sobre la necesaria para la neutralización de la muestra. La mezcla se agita durante 10 minutos a $70 \mathrm{rpm}$ y los jabones obtenidos se separan por decantación y filtración. Finalmente se eliminan las trazas de jabón por lavados sucesivos con agua.

La posterior decoloración de las muestras se realiza igualmente a $80^{\circ} \mathrm{C}$ bajo corriente de nitrógeno, añadiendo un $1 \%$ de tierra decolorante (GADOR C). La muestra se mantiene durante 15 minutos con agitación, tras lo cual el aceite se enfría y se filtra.
Finalmente, la muestra se desodoriza en sistema discontinuo de laboratorio. Se realizaron dos desodorizaciones para cada muestra a las temperaturas de 180 y $260{ }^{\circ} \mathrm{C}$ durante 3 y 2 horas respectivamente. La presión del sistema fue de 3 Torr.

\section{b) Refinación física}

Las condiciones de decoloración son similares a la utilizada en la refinación química con la excepción de la cantidad de tierra decolorante, que fue del 1,5\%. La desodorización de los aceites se realizó exclusivamente a $260^{\circ} \mathrm{C}$, durante 2 horas, ya que la temperatura de $180^{\circ} \mathrm{C}$ es insuficiente para la destilación de los ácidos grasos libres.

\section{DETERMINACIONES ANALITICAS}

a) Acidez libre, determinada segun NORMA UNE 55011.

b) Cuantificación de compuestos polares. Se sigue el método propuesto por la IUPAC para la determinación de la alteración en grasas de fritura, partiendo de un gramo de aceite (Waltking y Wessels, 1981).

c) Cuantificación de componentes menores glicerídicos. Se llevó a cabo mediante cromatografía líquida de exclusión (HPSEC) (Dobarganes et al., 1988). La fracción de compuestos polares obtenida como se indica en el punto anterior (b), es analizada en un cromatógrafo líquido Konik 500 A equipado con dos columnas de 100 y $500 \AA$ de PLgel de $30 \times 0.75 \mathrm{~cm}$ d.i. conectadas en serie. Se utilizó un detector de índice de refracción Hewlett Packard 1037 A. La fase móvil fue tetrahidrofurano a un flujo de $10 \mathrm{~mL} / \mathrm{min}$ y la concentración de la muestra de $\approx 15-20 \mathrm{mg} / \mathrm{mL}$ y la cantidad inyectada $10 \mu \mathrm{l}$.

\section{RESULTADOS Y DISCUSION}

La Tabla II recoge los resultados obtenidos en la evaluación de los componentes menores glicerídicos de los aceites de oliva sometidos a refinación en laboratorio. En primer lugar, es importante destacar que el objetivo de estas experiencias era analizar en detalle las modificaciones producidas como consecuencia del proceso. Para ello, se seleccionaron dos aceites vírgenes que poseen características muy diferentes. El primero era un aceite lampante con una elevada cantidad de ácidos grasos libres $(6,38 \%)$, mientras el segundo era un aceite corriente que no hubiera sido normalmente sometido al proceso de refinación. La principal diferencia entre las muestras iniciales radica en la cantidad de compuestos polares totales, muy elevada en la muestra primera como consecuencia de la cantidad de ácidos grasos libres y de diglicéridos originados simultáneamente en la hidrólisis de los triglicéridos. Existe igualmente una pequeña cantidad de dímeros, posiblemente dímeros oxidativos, atribuibles al largo periodo de almacenamiento del aceite ( $\approx 2$ años). Se ha incluido también en la Tabla los valores de la acidez libre expresada en $\mathrm{mg}$ de ácido oleico/g de grasa, así como el valor de la relación Diglicéridos/Acidos grasos (DG/Acidez). Esta rela- 
Tabla II

Cuantificación de componentes menores glicerídicos en aceites de oliva antes y después de ser refinados (mg/g)

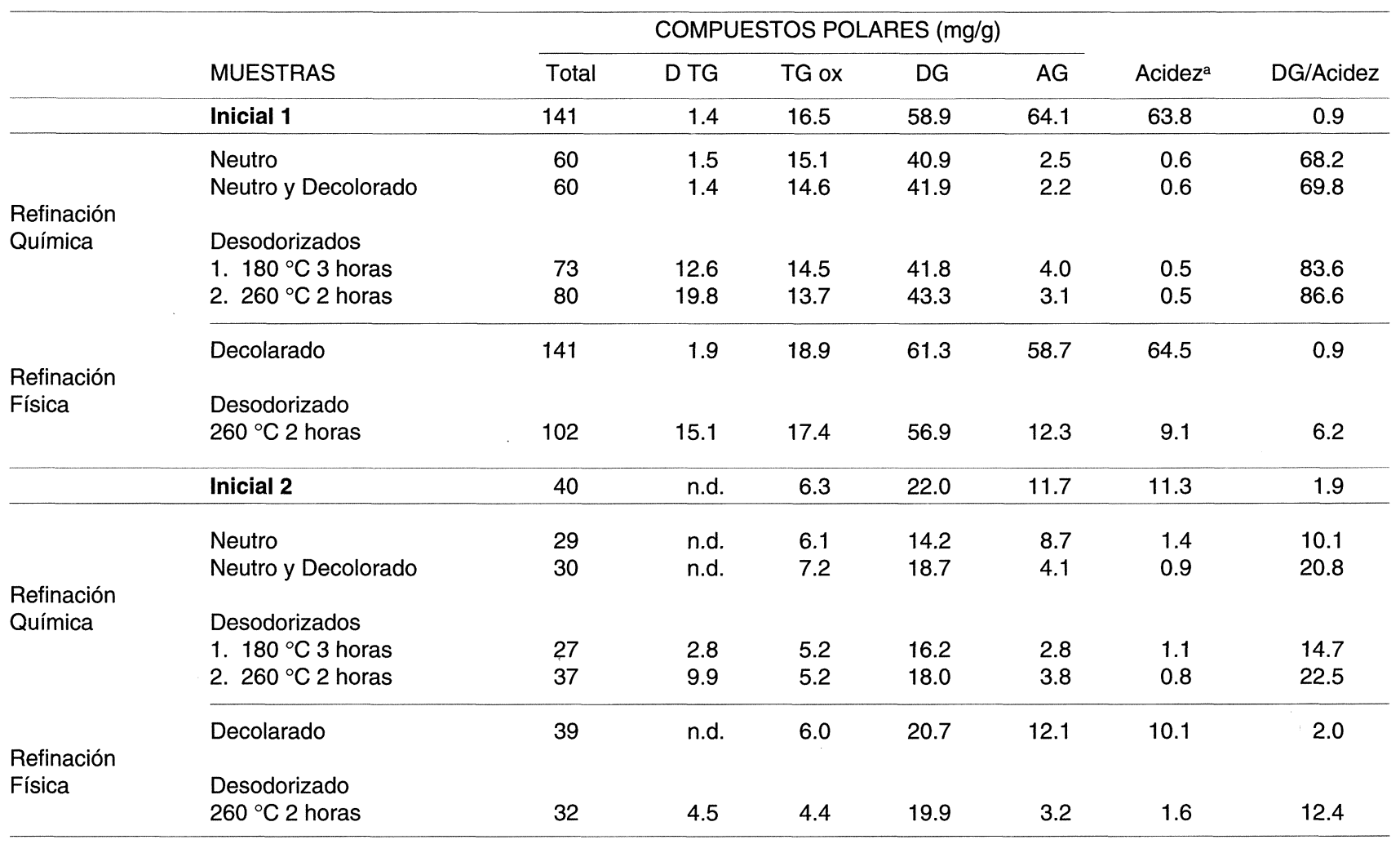

a: Expresada como mg de ácido oleico/g de aceite.

Abreviaturas: D TG: Dímeros de triglicéridos; TG ox: Triglicéridos oxidados; DG: Diglicéridos; AG: Acidos grasos; n.d.: no detectable.

ción se ha calculado a partir de las cantidades de diglicéridos y de la acidez libre, ya que la determinación de los ácidos grasos libres por cromatografía de exclusión está sobrevalorada por incluir aquellos compuestos del insaponificable que se encuentran en la fracción polar y tienen pesos moleculares similares a los ácidos grasos.

Como consecuencia de las distintas etapas del proceso de refinación, ambas muestras experimentan modificaciones de carácter general que se resumen en los siguientes puntos:

a) Como era de esperar, la neutralización disminuye la cantidad de compuestos polares como consecuencia, fundamentalmente, de la eliminación de ácidos grasos libres, aunque parece observarse también una disminución en la cantidad de digliceridos.

b) La formación de dímeros comienza a detectarse en la etapa de decoloración, aunque su incremento está fundamentalmente relacionado con la temperatura utilizada en la fase de desodorización. Por otra parte, los resultados obtenidos parecen indicar que la cantidad de dímeros de triglicéridos no sólo depende de la temperatura de desodorización sino también de la calidad inicial del aceite. Así, la cantidad encontrada es mucho más elevada, en todos los casos, en la muestra de elevada acidez aunque se han utilizado en las desodorizaciones los mismos valores de temperatura, tiempo, cantidad de vapor y presión. c) Los triglicéridos oxidados permanecen en todos los casos en los niveles iniciales debido a su baja volatilidad. Su cantidad en las muestras refinadas sería, por tanto, un buen indicador del estado inicial de oxidación del aceite virgen (Pérez-Camino et al., 1990).

d) La relación DG/Acidez aumenta significativamente en todos los casos como consecuencia de la disminución drástica de la acidez libre de las muestras. Teniendo en cuenta que una cantidad importante de aceites de oliva vírgenes se refinan debido a su alteración hidrolítica, los valores esperados de esta relación deben ser más elevados cuanto mayor sea la acidez inicial de las muestras. Como puede observarse en la Tabla II, el único valor encontrado para las muestras refinadas inferior a 10, es el correspondiente a la refinación física de la muestra de acidez elevada, lo que sin duda es debido a la cantidad de ácidos grasos libres $(0.91 \%)$ que todavía contiene la muestra.

De estos resultados se deduce que podrían definirse dos parámetros indicativos de la existencia del proceso de refinación: la presencia de dímeros en cantidades significativas y la relación DG/Acidez que se modifica sensiblemente debido a la eliminación de la mayor parte de los ácidos grasos libres.

Para conocer la validez de las conclusiones anteriores se procedió a la evaluación de 15 muestras de aceites de oliva vírgenes y refinados de origen garantizado, cuyos 
resultados se muestran en las Tablas III y IV. Como puede fácilmente observarse, no se detecta la presencia de dímeros en ninguno de los aceites vírgenes, mientras que se encuentran presentes en todas las muestras de aceites refinados, oscilando sus cantidades entre 2,2 y $14,4 \mathrm{mg} / \mathrm{g}$. Igualmente, las relaciones DG/Acidez son muy diferentes para ambos grupos de aceites, oscilando entre 1,5 y 4,4 para los vírgenes, y entre 11,5 y 32,9 para los refinados.

Por otra parte, en relación con los aceites de oliva virgen, puede observarse que, independientemente de los valores de acidez libre de los aceites, los componentes menores gliceridícos que se encuentran en mayor proporción son los digliceridos, que guardan una relación muy directa con la cantidad total de compuestos polares ( $r=$ 0.965).

Tabla III

Cuantificación de componentes menores glicerídicos en aceites de oliva virgen $(\mathrm{mg} / \mathrm{g})$.

\begin{tabular}{|c|c|c|c|c|c|c|c|}
\hline \multirow[b]{2}{*}{ MUESTRA } & \multicolumn{5}{|c|}{ COMPUESTOS POLARES } & \multirow[b]{2}{*}{ Acidez $^{a}$} & \multirow[b]{2}{*}{ DG/Acidez } \\
\hline & Total & D TG & TG ox & DG & $A G$ & & \\
\hline \multirow[t]{2}{*}{ V1 } & 33 & n.d. & 5.8 & 17.7 & 9.5 & 5.6 & 3.2 \\
\hline & & & $(17.7)^{\mathrm{b}}$ & $(53.6)$ & $(28.7)$ & & \\
\hline \multirow[t]{2}{*}{ V2 } & 35 & n.d. & 13.5 & 16.7 & 4.7 & 4.8 & 3.5 \\
\hline & & & $(38.6)$ & $(47.8)$ & $(13.6)$ & & \\
\hline \multirow[t]{2}{*}{ V3 } & 66 & n.d. & 7.6 & 32.4 & 25.9 & 19.6 & 1.6 \\
\hline & & & $(11.5)$ & $(49.1)$ & $(39.4)$ & & \\
\hline \multirow[t]{2}{*}{ V4 } & 49 & n.d. & 6.6 & 26.1 & 16.3 & 14.6 & 1.8 \\
\hline & & & $(13.5)$ & $(53.2)$ & (33.3) & & \\
\hline \multirow[t]{2}{*}{ V5 } & 72 & n.d. & 15.4 & 34.0 & 22.5 & 22.5 & 1.5 \\
\hline & & & $(21.3)$ & $(47.3)$ & $(31.4)$ & & \\
\hline \multirow[t]{2}{*}{ V6 } & 53 & n.d. & 10.8 & 28.2 & 14.0 & 8.8 & 2.0 \\
\hline & & & (20.4) & $(53.2)$ & (26.4) & & \\
\hline \multirow[t]{2}{*}{ V7 } & 35 & n.d. & 9.2 & 16.7 & 9.1 & 9.2 & 3.2 \\
\hline & & & $(26.2)$ & $(47.8)$ & $(26.0)$ & & \\
\hline \multirow[t]{2}{*}{ V8 } & 35 & n.d. & 6.9 & 19.2 & 8.8 & 7.2 & 2.1 \\
\hline & & & (19.8) & $(55.0)$ & $(25.2)$ & & \\
\hline \multirow[t]{2}{*}{ V9 } & 37 & n.d. & 11.2 & 17.8 & 7.8 & 7.4 & 2.4 \\
\hline & & & $(30.2)$ & " $(48.2)$ & (21.6) & & \\
\hline \multirow[t]{2}{*}{ V10 } & 34 & n.d. & 6.8 & 19.1 & 8.1 & 6.8 & 2.8 \\
\hline & & & (19.9) & (56.3) & (23.8) & & \\
\hline \multirow[t]{2}{*}{ V11 } & 44 & n.d. & 9.4 & 21.5 & 13.2 & 13.4 & 1.6 \\
\hline & & & (21.3) & $(48.7)$ & $(30.0)$ & ' & \\
\hline \multirow[t]{2}{*}{ V12 } & 32 & n.d. & 11.3 & 13.4 & 7.3 & 7.4 & 1.8 \\
\hline & & & $(35.4)$ & (41.9) & (22.7) & & \\
\hline \multirow[t]{2}{*}{$\mathrm{V} 13$} & 46 & n.d. & 13.6 & 19.4 & 13.0 & 11.5 & 1.7 \\
\hline & & & (29.6) & $(42.2)$ & (28.2) & & \\
\hline \multirow[t]{2}{*}{ V14 } & 35 & n.d. & 12.5 & 14.2 & 8.2 & 7.6 & 1.9 \\
\hline & & & $(35.8)$ & $(40.7)$ & (23.5) & & \\
\hline \multirow[t]{2}{*}{ V15 } & 72 & n.d. & 15.3 & 34.0 & 22.6 & 22.7 & 1.5 \\
\hline & & & $(21.3)$ & (47.3) & (31.4) & & \\
\hline
\end{tabular}

a: Expresada como $\mathrm{mg}$ de ácido oleico/g de aceite.

b: \% sobre Compuestos polares totales.

Abreviaturas en Tabla II.
En los aceites refinados (Tabla IV), la proporción de diglicéridos en el total de compuestos polares es aún más elevada debido, como se ha comentado, a la pérdida de la mayor parte de los ácidos grasos libres durante el proceso de refinación. El coeficiente de correlación con el contenido total de compuestos polares es igualmente elevado ( $r=0.943)$. Puede por tanto afirmarse que, tanto en los aceites vírgenes como refinados, el contenido en compuestos polares es tanto mayor cuanto más elevada es la alteración hidrolítica del aceite y, consecuentemente, menor su calidad.

Finalmente, la ausencia de dímeros en todas las muestras de oliva vírgenes analizadas contrasta con la encontrada en uno de los aceites vírgenes que fue sometido a refinación, lo que se atribuyó a la presencia de dímeros oxidativos, consecuencia del largo periodo de almacenamiento.

Tabla IV

Cuantificación de componentes menores glicerídicos en aceites de oliva refinados $(\mathrm{mg} / \mathrm{g})$.

\begin{tabular}{|c|c|c|c|c|c|c|c|c|}
\hline \multirow[b]{2}{*}{ MUESTRA } & \multicolumn{6}{|c|}{ COMPUESTOS POLARES } & \multirow[b]{2}{*}{ Acidez ${ }^{a}$} & \multirow[b]{2}{*}{ DG/Acidez } \\
\hline & Total & DTG & TG ox & DG & MG & $A G$ & & \\
\hline \multirow[t]{2}{*}{ R1 } & 80 & 6.8 & 10.8 & 57.7 & 1.1 & 3.5 & 1.8 & 32.0 \\
\hline & & $(8.5)^{b}$ & $(13.5)$ & $(72.2)$ & (1.4) & (4.4) & & $\because$ \\
\hline \multirow[t]{2}{*}{$\mathrm{R} 2$} & 55 & 3.5 & 20.9 & 25.7 & n.d. & 4.8 & 1.9 & 13.5 \\
\hline & & (6.4) & $(38.1)$ & $(46.7)$ & & (8.8) & & \\
\hline \multirow[t]{2}{*}{ R3 } & 57 & 8.1 & 17.8 & 28.0 & n.d. & 3.0 & 2.1 & 13.3 \\
\hline & & $(14.2)$ & $(31.3)$ & $(49.2)$ & & (5.3) & & \\
\hline \multirow[t]{2}{*}{ R4 } & 57 & 7.0 & 7.9 & 39.6 & n.d. & 5.5 & 2.0 & 18.3 \\
\hline & & $(12.3)$ & $(13.9)$ & $(64.2)$ & & (9.6) & & \\
\hline \multirow[t]{2}{*}{ R5 } & 44 & 3.7 & 6.9 & 28.8 & n.d. & 4.6 & 1.9 & 15.1 \\
\hline & & (8.4) & (15.7) & (65.4) & & (10.5) & & \\
\hline \multirow[t]{2}{*}{ R6 } & 65 & 6.7 & 8.1 & 43.7 & 1.6 & 4.7 & 3.5 & 12.5 \\
\hline & & (10.4) & (12.5) & (67.3) & (2.4) & (7.4) & & \\
\hline \multirow[t]{2}{*}{$\mathrm{R} 7$} & 65 & 7.5 & 15.7 & 38.9 & 0.3 & 2.5 & 1.4 & 27.8 \\
\hline & & $(11.6)$ & (24.1) & $(59.9)$ & $(0.5)$ & (3.9) & & \\
\hline \multirow[t]{2}{*}{ R8 } & 48 & 4.0 & 6.6 & 33.8 & n.d. & 3.5 & 1.9 & 17.8 \\
\hline & & (8.3) & $(13.8)$ & $(70.6)$ & & (7.3) & & \\
\hline \multirow[t]{2}{*}{$\mathrm{R} 9$} & 88 & 7.7 & 8.1 & 67.9 & 0.7 & 3.5 & 2.2 & 30.9 \\
\hline & & $(8.8)$ & (9.2) & (77.1) & $(0.8)$ & $(4.1)$ & & \\
\hline \multirow[t]{2}{*}{ R10 } & 31 & 2.2 & 5.9 & 19.4 & n.d. & 3.5 & 1.5 & 12.9 \\
\hline & & (7.1) & (19.2) & $(62.5)$ & & $(11.2)$ & & \\
\hline \multirow[t]{2}{*}{$\mathrm{R} 11$} & 49 & 4.7 & 8.5 & 32.9 & 0.3 & 2.5 & 1.8 & 18.3 \\
\hline & & (9.6) & (17.3) & $(67.2)$ & $(0.7)$ & (5.2) & & \\
\hline \multirow[t]{2}{*}{$\mathrm{R} 12$} & 44 & 12.8 & 7.8 & 19.6 & n.d. & 3.7 & 1.7 & 11.5 \\
\hline & & $(29.1)$ & (17.8) & $(44.6)$ & & (8.5) & & \\
\hline \multirow[t]{2}{*}{$\mathrm{R} 13$} & 35 & 14.4 & 6.5 & 12.6 & n.d. & 1.4 & 0.6 & 21.0 \\
\hline & & $(41.3)$ & (18.8) & $(35.9)$ & & $(4.0)$ & & \\
\hline \multirow[t]{2}{*}{$\mathrm{R} 14$} & 50 & 2.3 & 13.5 & 30.3 & n.d. & 3.9 & 1.7 & 17.8 \\
\hline & & (4.5) & $(27.0)$ & $(60.7)$ & & (7.8) & & \\
\hline \multirow[t]{2}{*}{$\mathrm{R} 15$} & 66 & 4.8 & 12.9 & 43.2 & n.d. & 5.0 & 2.2 & 19.6 \\
\hline & & (7.3) & (19.6) & $(65.5)$ & & (7.6) & & \\
\hline
\end{tabular}

a: Expresada como $\mathrm{mg}$ de ácido oleico/g de aceite.

b: \% sobre Compuestos polares totales.

Abreviaturas en Tabla II. 
Tabla $\mathrm{V}$

Cambios en los componentes menores glicerídicos de interés después de un año de almacenamiento ( $\mathrm{mg} / \mathrm{g}$ )

\begin{tabular}{|c|c|c|c|c|c|c|c|c|c|c|c|c|}
\hline \multirow[b]{2}{*}{ VARIEDAD } & \multirow{2}{*}{$\begin{array}{l}\text { ESTADO DE } \\
\text { MADUREZ }\end{array}$} & \multirow{2}{*}{$\begin{array}{l}\text { SISTEMA DE } \\
\text { EXTRACCION }\end{array}$} & \multicolumn{5}{|c|}{ COMPUESTOS POLARES INICIALES } & \multicolumn{5}{|c|}{ COMPUESTOS POLARES DESPUES DE 1 AÑO } \\
\hline & & & Total & D TG & $\mathrm{DG}$ & Acidez $^{\mathrm{a}}$ & DG/Acidez & Total & D TG & DG & Acidez $^{\mathrm{a}}$ & DG/Acidez \\
\hline \multirow{6}{*}{ PICUDA } & & - CENTRIFUGA & 23 & n.d. & 12.6 & 3.3 & 3.8 & 23 & 0.9 & 13.8 & 3.4 & 4.0 \\
\hline & VERDE & - PRENSA & 19 & n.d. & 12.5 & 4.6 & 2.7 & 24 & 0.7 & 17.2 & 4.5 & 3.8 \\
\hline & & & & & & & & & & & & \\
\hline & & - CENTRIFUGA & 26 & n.d. & 14.7 & 3.6 & 4.1 & 25 & 0.9 & 17.9 & 5.4 & 3.3 \\
\hline & MADURA & & & & & & & & & & & \\
\hline & & - PRENSA & 27 & n.d. & 17.7 & 5.3 & 3.3 & 30 & 0.9 & 23.6 & 6.7 & 3.5 \\
\hline \multirow{6}{*}{ PICUAL } & & - CENTRIFUGA & 19 & n.d. & 11.2 & 2.4 & 4.7 & 19 & 0.8 & 11.8 & 2.1 & 5.6 \\
\hline & VERDE & & & & & & & & & & & \\
\hline & & - PRENSA & 17 & n.d. & 10.2 & 3.0 & 3.4 & 20 & 0.7 & 17.9 & 3.9 & 4.6 \\
\hline & & - CENTRIFUGA & 19 & n.d. & 12.2 & 2.7 & 4.5 & 25 & 0.3 & 15.4 & 3.3 & 4.7 \\
\hline & MADURA & & & & & & & & & & & \\
\hline & & - PRENSA & 22 & n.d. & 14.2 & 3.4 & 4.2 & 27 & 0.5 & 16.3 & 4.9 & 3.3 \\
\hline \multirow{7}{*}{ MARTEÑA } & & - CENTRIFUGA & 19 & n.d. & 13.8 & 2.5 & 5.5 & 17 & 0.6 & 16.3 & 3.6 & 4.5 \\
\hline & VERDE & & & & & & & & & & & \\
\hline & & - PRENSA & 24 & n.d. & 15.3 & 3.6 & 4.2 & 25 & 0.9 & 18.0 & 5.7 & 3.2 \\
\hline & & & & & & & & & & & & \\
\hline & & - CENTRIFUGA & 37 & n.d. & 18.6 & 4.7 & 3.9 & 45 & 1.4 & 25.5 & 5.3 & 4.8 \\
\hline & MADURA & & & & & & & & & & & \\
\hline & & - PRENSA & 29 & n.d. & 16.0 & 3.4 & 4.7 & 50 & 1.0 & 18.4 & 4.9 & 3.7 \\
\hline
\end{tabular}

a: Expresada como $\mathrm{mg}$ de ácido oleico/g de aceite.

Abreviaturas en Tabla II.

Para comprobar este hecho, se realizó una experiencia de conservación con una serie de aceites vírgenes procedentes de tres variedades de aceitunas como se detalla en la parte experimental. La Tabla $\mathrm{V}$ resume los parámetros más significativos para las muestras recién obtenidas y después de un año de conservación. Como puede verse, independientemente de la variedad, del estado de madurez de las muestras, y del sistema de extracción utilizado, no se detecta la presencia de dímeros en los aceites recién obtenidos. Sin embargo, después de un año de almacenamiento a temperatura ambiente se detecta la presencia de dímeros de triglicéridos aunque en cantidades muy inferiores a las encontradas en los aceites refinados y compatibles con valores bajos de la relación DG/Acidez que no se modifica significativamente durante la conservación de los aceites.

En resumen, de los resultados de este estudio se deducen, entre otras, las siguientes conclusiones:

a) El contenido total en componentes menores glicerídicos está inversamente relacionado con la calidad del aceite de oliva virgen o refinado (y directamente relacionado con el contenido en diglicéridos).

b) La ausencia de compuestos de polimerización es la característica de mayor interés para la identificación de los aceites de oliva vírgenes.

c) El proceso de refinación introduce modificaciones apreciables en los componentes menores glicerídicos. La presencia de dímeros de triglicéridos y la elevada relación DG/Acidez son características principales de los aceites refinados.

\section{AGRADECIMIENTO}

Los autores quieren expresar su agradecimiento a la CICYT (Proyecto ALI88-0208) y a la Junta de Andalucía por la financiación aportada. $A D^{\mathrm{a}} \mathrm{M}$. Giménez y M. Serrano por la ayuda prestada.

\section{BIBLIOGRAFIA}

Amelotti, G. (1987).- "Modificazioni indotte de processo di raffinazione fioca nella struttura dei trigliceridi dell'olio di oliva".- Riv. Ital. Sostanze Grasse 64, 223-226.

Boletín Oficial del Estado ${ }^{\circ} 44$ de 21 de Febrero de 1983.

Diario Oficial de las Comunidades Europeas № $L 248$ de 5 de Septiembre de 1991.

Dobarganes, M.C., Pérez-Camino, M.C. y Márquez-Ruiz, G. (1988).- "High performance size exclusion chromatography of polar compounds in heated and non-heated fats".- Fat Sci. Technol. 90, 308-311.

Eder, S.R. (1982).- "Über die Bildung von Artefakten bei der Dämpfung von Speiseölen und -fetten".- Fette Seifen Anstriechmittel 84, 136-141.

Faur, L. (1989a). - "Influence des traitements de raffinage et de transformation sur la qualité et la stabilité des corps gras. (première partie)".- Rev. Fr. Corps Gras 36, 265-270.

Faur, L. (1989b). - "Influence des traitements de raffinage et de transformation sur la qualité et la stabilité des corps gras. (Deuxième partie).- Rev. Fr. Corps Gras 36, 265-270.

Firestone, D. (1987).- "Control of olive oil adulteration and misbranding in the United Sates".- Riv. Ital. Sostanze Grasse 64, 293-297.

Gomes, T. y Catalano, M. (1988).- "Caratteri di qualita degli oli alimentari. I: trigliceride dimeri".- Riv. Ital. Sostanze Grasse 65, 125-127.

Gracián, J. y Mancha, M. (1971).- "Fenómenos de transesterificación durante el calentamiento de aceites de oliva y orujo y su influencia en la alteración de la estructura glicerídica".- Grasas y Aceites 22, 30-39.

Johansson, A. (1979).- "The effect of processing on the content and composition of free sterols and sterol esters in soybean oil".- J. Am. Oi Chemists' Soc. 56, 886-889. 
Jung, M.Y., Yoon, S.H. y Min, D.B., (1989).- "Effects of processing steps on the contents of minor compounds and oxidation of soybean oil".- J. Am. Oil Chemists' Soc. 66, 118-120.

Kapoulas, V.M. y Andrikopoulos, N.K. (1987).- "Detection of virgin olive oil adulteration with refined oils by second derivative spectrophotometry".Food Chem. 23, 183-192.

Kiritsakis, A.K. (1991).- "Olive Oil”. Ed. American Oil Chemists' Society.Champaing, IL, USA

Kochlar, S.P. (1983).- "Influence of processing on sterols of edible vegetable oils".- En: Prog. Lipid Res. Vol. 22, p.161-188.- Pergamon press Ltd., Gran Bretaña.

Lanzón, A., Cert, A. y Albi, T. (1989).- "Detección de la presencia de aceite de oliva refinado en el aceite de oliva virgen".- Grasas y Aceites 40, 385-388.

Norma UNE 55-011.- "Determinación de la acidez libre".

Organización de las Naciones Unidas para la Alimentación y la Agricultura (F.A.O.), Instituto de Investigaciones Agrarias (I.N.I.A.) y Ministerio de Agricultura de España. (1975).- "Manual de Elaiotecnia".- Ed. Agrícola Española, Madrid.

Pérez-Camino, M.C., Márquez-Ruiz, G., Ruiz-Méndez, M.V. y Dobarganes, M.C. (1990).- "Determinación cuantitativa de triglicéridos oxidados para la evaluación global del grado de oxidación en aceites y grasas comestibles".- Grasas y Aceites 41, 366-370.

Taponeco, G. y Giaconi, V. (1970).- "Struttura gliceridica degli oli d'oliva e sue variazioni nel corso della raffinazione".- Riv. Ital. Sostanze Grasse 47, 856-860.

Tisconia, E., Forina, M. y Evangelisti, F. (1982).- "Composizione chimica dell'olio di oliva e sue variazioni in dotte dal processo di rettificazione".Riv. Ital. Sostanze Grasse 59, 519-556.

Waltking, A.E. y Wessels, H. (1981).- "Chromatographic separation of polar and nonpolar components of frying fats".- J. Assoc. Off. Anal. Chem. 64, 1329-1330.

(Recibido: Enero 1993) 